\title{
Estratégias atuais utilizadas para o ensino da empatia na graduação médica: revisão sistemática
}

\author{
Current strategies used for teaching empathy in medical graduation: systematic review \\ Estrategias actuales utilizadas para enseñar empatía en graduación médica: revisión \\ sistemática
}

Eliane Perlatto Moura ${ }^{1 *}$, Tatiana Perlatto Moura², Júlia Coutinho Cordeiro¹, Thayná de Freitas Chaves $^{1}$, Aline Barbosa Peixoto ${ }^{1}$, José Maria Peixoto ${ }^{1}$.

\section{RESUMO}

Objetivo: Realizar uma revisão sistemática da literatura sobre estratégias atuais utilizadas para o ensino da empatia na graduação médica. Métodos: Pesquisou-se artigos nas bases de dados PubMed, Scielo e Eric. Descritores: (Empathy) AND (Teaching or academic training or educational technic or educational technics or teaching method or teaching methods) AND (Medical student or medical students or student, medical). Foram incluídos artigos publicados em inglês ou português; a partir de 2016; com estudantes de medicina; e palavra "Empatia" no título. Os dados foram extraídos por dois revisores de forma independente, considerando-se: nome do artigo, ano/ país da publicação, amostra, intervenção utilizada, instrumento de avaliação da empatia, resultados e conclusão. Resultados: 16 artigos selecionados classificados em: "Arte" - teatro, literatura, cinema e interpretação de imagens; "Reflexão" atividades reflexivas grupais ou individuais sobre uma consulta médica; "Curso de Comunicação" incluiu cursos ou workshops sobre comunicação empática; "Interação com o Paciente" interação com pacientes reais, virtuais, atores ou por meio de role-play; e "Tecnologia" com estratégias virtuais. Considerações Finais: A empatia é passível de ser ensinada, sendo as estratégias mais efetivas aquelas que abordam os aspectos emocionais e cognitivos. Métodos de ensino combinados e longitudinais sugerem ser mais benéficos para a consolidação do aprendizado.

Palavras-chave: Empatia, Estudantes de medicina, Educação médica, Ensino.

\section{ABSTRACT}

Objective: To carry out a systematic review of the literature on current strategies used for teaching empathy in medical graduation. Methods: Articles were searched in the PubMed, Scielo and Eric databases. Descriptors: (Empathy) AND (Teaching or academic training or educational technic or educational technics or teaching method or teaching methods) AND (Medical student or medical students or student, medical). Articles published in English or Portuguese were included; from 2016; with medical students; and the word "Empathy" in the title. The data were extracted by two reviewers independently, considering: name of the article, year / country of publication, sample, intervention used, empathy assessment instrument, results and conclusion. Results: 16 articles selected and classified in: "Art"- theatre, literature, cinema and image interpreting; "Reflection" group or individual reflective activities about a medical consultation; "Communication Course" included courses or workshops on empathic communication; "Interaction with the Patient" interaction with real patients, virtual patients, actors or through role-play; and "Technology" with virtual strategies. Conclusion: Empathy can be taught, with the most effective strategies being those that address emotional and cognitive aspects. Combined and longitudinal teaching methods suggest that they are more beneficial for the consolidation of learning.

Keywords: Empathy, Medical students, Medical education, Teaching.

\section{RESUMEN}

Objetivo: Realizar una revisión sistemática de la literatura sobre las estrategias actuales de enseñanza de la empatía en la graduación médica. Métodos: Se realizaron búsquedas de artículos en las bases de datos PubMed, Scielo y Eric. Descriptores: (Empatía) Y (Docencia o formación académica o técnica educativa o técnica educativa o método de enseñanza o métodos de enseñanza) Y (Estudiante de medicina o estudiantes de medicina o estudiante de medicina). Se incluyeron artículos publicados en inglés o portugués; desde 2016;

1 Universidade José do Rosário Vellano, Belo Horizonte - MG. *E-mail: elianeperlatto@gmail.com

2 Universidade Federal de Minas Gerais, Belo Horizonte - MG. 
con estudiantes de medicina; y la palabra "Empatía" en el título. Los datos fueron extraídos por dos revisores de forma independiente, considerando: nombre del artículo, año / país de publicación, muestra, intervención utilizada, instrumento de evaluación de la empatía, resultados y conclusión. Resultados: 16 artículos seleccionados clasificados en: "Arte" - teatro, literatura, cine e interpretación de imágenes; "Reflexión" grupal o actividades de reflexión individual sobre una consulta médica; El "Curso de comunicación" incluyó cursos o talleres sobre comunicación empática; Interacción con la paciente interacción con pacientes reales, pacientes virtuales, actores o mediante juegos de roles; y "Tecnología" con estrategias virtuales. Conclusión: Se puede enseñar la empatía, siendo las estrategias más efectivas aquellas que abordan aspectos emocionales y cognitivos. Los métodos de enseñanza combinados y longitudinales sugieren que son más beneficiosos para la consolidación del aprendizaje.

Palabras clave: Empatía, Estudiantes de medicina, Educación médica, Enseñanza.

\section{INTRODUÇÃO}

No dicionário empatia é definida como: "A capacidade psicológica para se identificar com o eu do outro, conseguindo sentir o mesmo que este nas situações e circunstâncias por esse outro vivenciadas" (FERREIRA $A B, 2002)$. Entretanto, o conceito de empatia é complexo e multidimensional sendo a capacidade de um indivíduo sentir o que o outro sente, ou de se imaginar no lugar do outro. Ainda, a empatia pode ser descrita como a habilidade de compreender o contexto de outra pessoa (JEFFREY D, 2016; RATKA A, 2018). Assim, a empatia permite compreender a perspectiva do próximo, fazendo com que este sinta-se acolhido (COTTA FCK, 2020; BATISTA NA, 2019).

$\mathrm{Na}$ empatia existem dois componentes: um afetivo e outro cognitivo. No componente afetivo o observador compartilha os estados emocionais dos outros (neurônios espelhos) de modo inconsciente estando sujeito a um processo de habituação sensorial, caracterizado por redução destas sensações após exposições repetidas à mesma situação (CAMPBELL T, 2019).

O componente cognitivo refere-se à habilidade de identificar, entender e explicitar a resposta empática ao outro, de modo a fornecer ajuda (JEFFREY D, 2016). Portanto, apesar da presença inata da empatia afetiva ser relevante, autores sugerem que, no âmbito da educação médica, a empatia cognitiva seria a mais importante e aquela possível de ser ensinada (LAUGHEY W, 2019; PEIXOTO JM, 2020; KLEINSMITH A, 2015).

A empatia é essencial para promover um cuidado completo e integralizado ao paciente de forma humanizada. Ela ajuda a aumentar os resultados terapêuticos e a qualidade de vida do paciente, além de auxiliar o próprio médico a aumentar a acurácia diagnóstica e a promover um maior bem-estar durante os atendimentos, implicando em maior satisfação dos profissionais de saúde e dos pacientes (ARISO JM, 2018). Assim, o médico empático é capaz de entender os aspectos subjetivos do adoecimento, sendo apto a consolidar uma relação médico-paciente de confiança (COTTA FCK, 2020; BATISTA NA, 2019; SAN-MARTÍN M, 2017; CHEN AK, 2016).

Contudo, nota-se que, com o avançar do curso, há uma queda nos níveis de empatia dos estudantes de medicina. O declínio inicia-se, principalmente, durante a transição do ciclo básico para o clínico. Atribui-se à essa queda a alta demanda de atendimentos, um currículo desafiador e uma rotina estressante. Nesse sentido, são necessárias estratégias que preservem ou aumentem a empatia do estudante médico durante sua formação (PEIXOTO JM, 2020; GRAHAN, 2016; SON D, 2018; CHEN A, 2018).

Visto que a empatia é essencial dentro da educação médica, estudos têm demonstrado diversas formas de ensiná-la. $O$ ensino dessa habilidade abrange desde o treinamento de comunicação tradicional até o envolvimento da arte e da tecnologia. Nesse sentido, para o aprimoramento da formação do futuro profissional médico, é necessário que estratégias de ensino sejam consolidadas de maneira a favorecer 0 desenvolvimento da empatia durante a graduação (BATISTA NA, 2019; KATAOKA H, 2019; AHMADZADEH $A, 2019)$. Este estudo tem como objetivo realizar uma revisão sistemática da literatura sobre os métodos que estão sendo utilizados para o ensino da empatia. 


\section{MÉTODOS}

Revisão sistemática da literatura, conduzida conforme recomendações da Cochrane Collaboration (HIGGINS JPT, 2011) na qual foram revisados artigos publicados originalmente em inglês e português, que utilizaram alguma estratégia para o ensino da empatia.

\section{Construção da pergunta de pesquisa}

Para elaboração da pergunta foi utilizada a estratégia PICo, sendo "P" população - estudantes de medicina; "I" fenômeno de interesse - estratégia de ensino da empatia e "Co" contexto - graduação médica (CARDOSO V, 2019).

\section{Seleção dos estudos e critérios de inclusão e exclusão}

Primeiramente, realizou-se busca online nas bases de dados PubMed, Scielo e Eric, com os descritores: (Empathy) AND (Teaching or academic training or educational technic or educational technics or teaching method or teaching methods) AND (Medical student or medical students or student, medical) (Quadro 1).

Quadro 1 - Estratégia de busca utilizada para a pesquisa nas bases de dados

\begin{tabular}{|c|c|}
\hline Número da estratégia & Descritores \\
\hline 1 & $\begin{array}{r}\text { Teaching or academic training or educational technic or educational technics or } \\
\text { teaching method or teaching methods }\end{array}$ \\
\hline 2 & Medical student or medical students or student, medical \\
\hline
\end{tabular}

Legenda: 1- Descritores relativos à estratégia de ensino da empatia (I da estratégia PICO); 2- Descritores relativos à população ( $\mathrm{P}$ da estratégia $\mathrm{PICO})$

Fonte: Moura EP, et al., 2021.

Os critérios de inclusão foram: artigos em língua inglesa ou em português com a palavra "Empatia" no título, publicados a partir de 2016 e com população - estudante de medicina em graduação. Foram excluídos os artigos de revisão; publicações de doutorado ou mestrado e estudos em cenários, que não o da graduação médica.

\section{Qualidade dos estudos}

Os artigos selecionados foram submetidos a uma avaliação de qualidade por dois autores de forma independente e pontuados levando-se em consideração: população estudada; intervenção; processo de coleta de dados; método analítico; resultados e conclusão (1 ponto cada) (MAIROT LTS, 2019). Foi atribuída uma pontuação a cada um dos estudos, que variou de 0 a 6 . A qualidade de cada artigo foi classificada como inadequado (1-3) e adequado (4-6). Foram considerados aptos a serem incluídos os artigos que apresentaram classificação adequada, sendo eliminados os artigos que não pontuaram no critério intervenção.

\section{Processo de coleta e extração de dados}

Os dados foram extraídos de maneira padronizada pelos revisores, que trabalharam independentemente e levaram em consideração as seguintes variáveis: autor, nome do artigo, ano e país da publicação, amostra, intervenção utilizada, instrumento de avaliação da empatia, resultados e conclusão.

\section{RESULTADOS}

\section{Seleção dos estudos}

Inicialmente foram identificados 212 artigos. Após eliminação dos estudos realizados em outro contexto que não o da graduação médica, foram selecionados 25 artigos para revisão de texto completo. Destes, nove foram excluídos por obter pontuação baixa na avaliação de qualidade ou por terem recebido nota zero no critério intervenção. Ao final, 16 artigos foram selecionados para compor a amostra. Dentre esses, três artigos (19\%) receberam a nota quatro, dois artigos (13\%) pontuaram 5 pontos e 11 artigos (68\%) pontuaram 6 pontos (Figura 1). 
Figura 1 - Etapa da seleção dos estudos.

Estudos identificados nas bases de dados: Pubmed, SciELO e Eric; $(\mathbf{n}=\mathbf{2 1 2})$
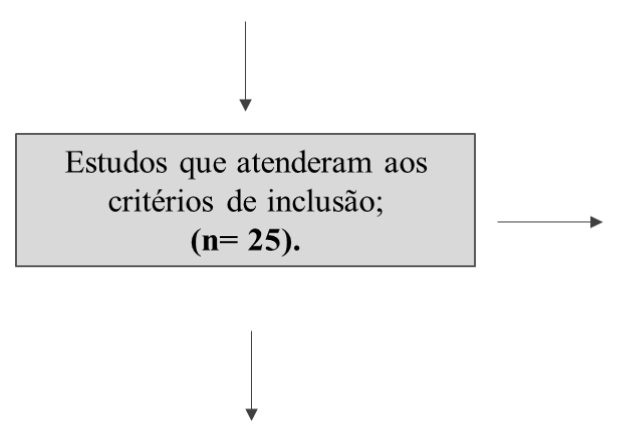

\section{Estudos selecionados para} compor amostra $(n=16)$.
Critérios de inclusão: artigos originais em lingua inglesa ou em português; publicados a partir de 2016; população estudada - estudante de medicina em graduação e artigos que continham a palavra "Empatia" no título.
Descritores: Empathy AND Teaching or academic training or educational technic or educational technics or teaching method or teaching methods AND Medical student or medical students or student, medical.
Avaliação de qualidade por meio dos tópicos: população estudada; intervenção; processo de coleta de dados; método analítico; resultados e conclusão, valendo 1 ponto cada. Pontuação variou de 0 a 6 , para cada estudo.
Estudos excluídos:

- Classificação inadequada (1- 3 pontos);

- Artigos com pontuação 0 no critério " intervenção". $(\mathbf{n}=9)$

Fonte: Moura EP, et al., 2021.

\section{Características do estudo}

Os artigos selecionados foram publicados entre janeiro de 2016 e março de 2020 , sendo quatro publicados em 2016, seis em 2017, dois em 2018, quatro em 2019 e um em 2020. Os primeiros autores dos artigos eram de instituições situadas no Japão $(n=1)$, Índia $(n=1)$, Alemanha $(n=1)$, Espanha $(n=1)$, Irã $(n=1)$, Reino Unido $(n=1)$, Israel $(n=2)$, Brasil $(n=2)$ e Estados Unidos $(n=6)$. Todos os artigos analisados constituíram-se de estudos experimentais, e o número total de sujeitos abordados nos estudos variou de 20 a 343.

\section{Intervenções utilizadas para ensinar empatia}

Optou-se, neste estudo, por categorizar as intervenções utilizadas para o ensino da empatia em: Arte; Reflexão; Curso de Comunicação; Interação com o Paciente e Tecnologia; com intuito de facilitar o entendimento dos métodos de forma didática.

Na categoria "Arte" foram incluídos estudos que utilizaram teatro, literatura, cinema, interpretação de imagens e integração da arte em cursos de humanidades médicas. Na categoria "Reflexão" foram incluídos estudos que tinham como proposta discussão ou reflexão do aluno durante uma consulta médica. $\mathrm{Na}$ categoria "Curso de Comunicação" inclui-se estudos que realizaram cursos de comunicação e/ou humanidades, ou workshops. A categoria "Interação com o paciente" foi composta por estudos que utilizaram: pacientes reais; role-play; atores e pacientes virtuais. Na categoria "Tecnologia" foram incluídos estudos que utilizaram vídeos de consultas, programas e jogos criados especificamente para ensino da empatia.

A duração das intervenções variou em: intervenção única $(n=8)$, quando foi realizado apenas um encontro; intervenções múltiplas $(n=5)$, com múltiplos encontros dentro de um mesmo semestre; intervenções longitudinais $(n=2)$, quando as intervenções em mais de um momento do curso. As intervenções foram realizadas majoritariamente durante os primeiros quatro anos do curso $(n=15)$.

\section{Ensino da empatia utilizando artes}

Em dois estudos, os alunos foram submetidos a cursos de humanidades envolvendo interpretação de artes visuais (GRAHAM J, 2016; BENTWICH ME, 2017) e literatura (GRAHAM J, 2016). Para Bentwich ME e Gilbey P (2017), a análise de diferentes compreensões de uma mesma arte, por meio do método Estratégias de Pensamento Visual (Visual Thinking Strategies - VTS), contribui para a melhoria da empatia dos estudantes. 
Graham J, et al. (2016) reforçam que a interpretação de artes visuais e literatura pode gerar resultados favoráveis no aumento da empatia ao estimular os alunos a enxergarem uma mesma situação por diferentes pontos de vista. Shapiro J, et al. (2019) discutem a utilização de cursos de humanidades envolvendo aulas de teatro e práticas de improvisação, como ferramenta de ensino, especialmente para estudantes com baixos níveis de empatia. Ahmadzadeh A, et al. (2019) observaram que filmes podem levar a melhoria da empatia, especialmente quando combinados com outros métodos de ensino, pois estimulam um envolvimento emocional e identificação com os personagens.

\section{Ensino da empatia utilizando reflexão}

Um dos fatores que compõem a habilidade empática é a tomada de perspectiva, isto é, a capacidade de compreender a perspectiva e os sentimentos de outra pessoa (SUARTZ CV, 2013). Diante disso, Peixoto JM e Moura EP (2020), D'souza PC, et al. (2019) e Schweller M, et al. (2017) solicitaram aos alunos que refletissem sobre as perspectivas, pensamentos e emoções do paciente como estratégia para 0 desenvolvimento da empatia.

\section{Ensino da empatia utilizando cursos de comunicação}

Foram incluídos, nessa categoria, artigos que tiveram como intervenção cursos com o objetivo de treinar habilidades de comunicação, de maneira a torná-la mais empática (KATAOKA H, 2019; AHMADZADEH A, 2019; D'SOUZA PC, 2019; KAPLAN-LISS E, 2018; MEIROVICH A, 2016; RUIZ-MORAL R, 2017; WÜNDRICH $M, 2017)$, e aqueles que apresentaram, para o ensino da empatia, uma abordagem multidisciplinar, em que pelo menos uma das estratégias abordavam o treinamento de comunicação na relação médico-paciente (AHMADZADEH A, 2019; SHAPIRO J, 2019; SCHEWELLER, 2017).

Nesse sentido, a maioria das intervenções não consiste apenas no curso de comunicação, mas sim em métodos de ensino integrativos que perpassam a arte, a tecnologia, a interação com o paciente e a reflexão. Sete artigos envolveram o curso de comunicação integrado com algum tipo de interação com o paciente (KATAOKA H, 2019; SHAPIRO J, 2019; D'SOUZA PC, 2019; SCHEWELLER, 2017; KAPLAN-LISS E, 2018; RUIZ-MORAL R, 2017; WÜNDRICH M, 2017); quatro artigos tiveram o curso de comunicação em interdisciplinaridade com o uso da tecnologia (SHAPIRO J, 2019; D'SOUZA PC, 2019; SCHEWELLER, 2017; MEIROVICH A, 2016); dois artigos integraram o curso de comunicação com a habilidade de reflexão (D'SOUZA PC, 2019; SCHEWELLER, 2017), e um estudo integrou o uso da arte com curso de comunicação (AHMADZADEH A, 2019).

Ahmadzadeh A, et al. (2019) ressaltam que a utilização de métodos combinados pode ser benéfica para o aumento da empatia, ao demonstrarem que assistir filme juntamente com workshop de habilidades de comunicação foi mais efetivo para aumentar a empatia do que as intervenções realizadas isoladamente. Segundo Kaplan-Liss E, et al. (2018), curso de comunicação com exercícios de improvisação utilizando exercícios verbais e não verbais, palestras, discussões, role-play e entrevistas filmadas, pode ser um modelo eficaz para ensinar os alunos a se comunicarem com empatia.

Shapiro J et al. (2019) observaram que a abordagem da compaixão e empatia no contexto clínico através de palestras e interações com paciente, resultou em melhoria significativa da empatia autorreferida, particularmente entre os estudantes com pontuação inicial baixa de empatia e manutenção dos níveis de empatia naqueles com pontuação alta durante o primeiro ano clínico de treinamento. Para Schweller M, et al. (2017), o contato precoce dos estudantes do primeiro ano da graduação com pacientes reais é fundamental para o desenvolvimento da empatia e da relação médico-paciente.

Estudos realizados por Meirovich A, et al. (2016), Ruiz-Moral R, et al. (2017), Kataoka H, et al. (2019), e Wündrich M, et al. (2017) observaram que a utilização de paciente simulados seguido de análise de vários aspectos da comunicação é uma estratégia eficaz para o desenvolvimento da empatia nos estudantes. Para D'souza PC, et al. (2019), a identificação de técnicas corretas e incorretas de comunicação empática por meio de videoclipes projetados, interações simuladas com os pacientes e de role-plays com professores como pacientes simulados, seguidos de reflexão sobre os pensamentos e emoções, associou-se à melhora significativa nos níveis empáticos imediatamente após a intervenção e uma queda menor a longo prazo. Estes autores enfatizam a necessidade de intervenções recorrentes ao longo do currículo médico. 
Vários autores destacam a importância de discussões em grupo para o desenvolvimento da empatia nos estudantes (SHAPIRO J, 2019; SCHEWELLER, 2017; SWEENEY K, 2018). Meirovich A, et al. (2016) ressaltaram que a relação estudante-tutor tem papel fundamental na aprendizagem e que 0 treinamento pedagógico dos tutores com ênfase na importância dos conhecimentos, habilidades, atitudes e crenças trazidos pelos alunos para o ambiente educacional, tem correlação com a melhoria no comportamento empático dos estudantes.

\section{Ensino da empatia utilizando tecnologia}

Chen A, et al. (2018), utilizou o videogame "That Dragon, Cancer", que permite ao jogador vivenciar o cotidiano e os sentimentos da família de um menino diagnosticado com câncer. $O$ impacto emocional desencadeado pelo jogo torna-o uma estratégia promissora para o ensino da empatia em estudantes de medicina. Foster A, et al. (2016) empregaram simulações de pacientes virtuais realizadas por computador para o ensino da empatia. Shapiro J et al. (2019) utilizaram o Google Class para gravar um encontro entre aluno e paciente, que posteriormente era analisado pelos alunos e pelo mentor.

\section{Ensino da empatia utilizando interação com pacientes}

\section{Paciente real}

Três artigos utilizaram depoimentos de pacientes reais a respeito de suas experiências com o sistema de saúde e de suas interações com médicos para o ensino da empatia (SCHWELLER M, 2017; SWEENEY K, 2018; HENDRIKSZ T, 2016). Schweller M, et al. (2017) e Hendriksz T (2016) relataram que mostrar aos alunos a percepção dos pacientes acerca de suas doenças é uma estratégia eficaz para o ensino da empatia. Sweeney K e Baker $\mathrm{P}$ (2018), observaram resultados semelhantes ao utilizarem gravações de depoimentos dos pacientes acerca de suas experiências hospitalares. A sensibilização e reflexão dos alunos com o relato dos pacientes promoveu aumento dos comportamentos empáticos.

\section{Pacientes simulados (atores)}

Quatro estudos (KATAOKA H, 2019; RUIZ-MORAL R, 2017; WÜNDRICH M, 2017; FOSTER A, 2016) organizaram entrevistas médicas com pacientes simulados com o intuito de avaliar a interação aluno-paciente, as habilidades de comunicação e atitudes empáticas adquiridas durante cursos de comunicação. Estes autores observaram que a utilização de pacientes simulados é uma estratégia eficaz para o ensino da empatia durante a graduação.

\section{Pacientes Virtuais}

A utilização de pacientes virtuais por meio do uso de softwares para promover a interação com os estudantes e desenvolver a comunicação, visando o treinamento empático, foi uma estratégia considerada promissora (SHAPIRO J, 2019; FOSTER A, 2016).

\section{Role-play}

Três estudos utilizaram a estratégia de Role-play para o ensino da empatia (D'SOUZA PC, 2019; KAPLANLISS E, 2018; RUIZ-MORAL R, 2017). Kaplan-Liss E, et al. (2018) afirmaram que a utilização do role-play possibilitou aprimorar a construção das interações médico-paciente e as habilidades de comunicação. RuizMoral R, et al. (2017) utilizaram essa ferramenta buscando ensinar aos alunos uma percepção ampla do processo de adoecimento dos pacientes e assim, possibilitar o aumento das atitudes empáticas durante as consultas.

\section{Avaliação dos níveis de empatia dos estudantes}

Para a avaliação da empatia, observou-se que oito artigos formularam questionários próprios com escala Likert, um artigo usou o Sistema de Codificação de Comunicação Empática (ECCS); dois artigos utilizaram Patient-Provider Orientation Scale (PPOS); sete artigos usaram abordagens qualitativas para avaliar a empatia baseadas em feedbacks e narrativas dos alunos e avaliadores; nove artigos utilizaram a Escala de Jefferson para Empatia (JSE). No quadro 2, estão as características dos estudos analisados. 
Quadro 2 - Características dos 16 estudos analisados acerca das estratégias de ensino da empatia na educação médica, identificados na literatura e publicados entre 2016 a 2020.

\begin{tabular}{|c|c|c|c|c|c|c|c|}
\hline Autor e Título & $\begin{array}{l}\text { Ano e país } \\
\text { da } \\
\text { publicação }\end{array}$ & $\begin{array}{l}\text { Participantes } \\
\text { do estudo }\end{array}$ & $\begin{array}{l}\text { Intervenção } \\
\text { opcional } \\
\text { /obrigatória }\end{array}$ & $\begin{array}{l}\text { Duração da } \\
\text { intervenção }\end{array}$ & Intervenção/Tipo da intervenção* & $\begin{array}{l}\text { Instrumento de } \\
\text { medida/ avaliação } \\
\text { da empatia }\end{array}$ & $\begin{array}{c}\text { Autores reportaram um } \\
\text { aumento significativo da } \\
\text { empatia? }\end{array}$ \\
\hline $\begin{array}{c}\text { Bentwich ME e } \\
\text { Gilbey P (2017). } \\
\text { More than visual } \\
\text { literacy: art and } \\
\text { the enhancement } \\
\text { of tolerance for } \\
\text { ambiguity and } \\
\text { empathy. }\end{array}$ & $\begin{array}{l}2017 \\
\text { Israel }\end{array}$ & $\begin{array}{c}67 \\
\text { estudantes } \\
\text { do } 1^{\circ} \text { ano do } \\
\text { curso de } \\
\text { medicina }\end{array}$ & Obrigatória & $\begin{array}{l}\text { Uma única } \\
\text { intervenção de } 90 \\
\text { minutos }\end{array}$ & $\begin{array}{l}\text { Utilizou-se a intervenção Visual } \\
\text { Thinking Strategies (VTS). Nela os } \\
\text { alunos deveriam interpretar e } \\
\text { responder perguntas sobre } 5 \text { imagens } \\
\text { famosas que envolviam situações de } \\
\text { adoecimento, falecimento e de } \\
\text { pacientes mortos. As perguntas foram: } \\
\text { (1) "O que está acontecendo na } \\
\text { imagem exibida?", (2) "O que você vê } \\
\text { na imagem que te faz pensar isso?" e } \\
\text { (3) Tente achar mais detalhes } \\
\text { interessantes na imagem. Depois, } \\
\text { houve uma discussão de } 15 \text { minutos. }\end{array}$ & $\begin{array}{l}\text { Instrumento do } \\
\text { próprio autor }\end{array}$ & $\begin{array}{l}\text { Sim, principalmente de } \\
\text { maneira indireta, ao } \\
\text { aumentar a tolerância } \\
\text { à ambiguidade }\end{array}$ \\
\hline $\begin{array}{c}\text { Graham J, et al. } \\
(2016) \\
\text { Medical } \\
\text { Humanities } \\
\text { Coursework Is } \\
\text { Associate with } \\
\text { Greater Measured } \\
\text { Empathy in } \\
\text { Medical Students. }\end{array}$ & $\begin{array}{l}2016 \\
\text { EUA }\end{array}$ & $\begin{array}{c}68 \\
\text { estudantes } \\
\text { de medicina }\end{array}$ & Opcional & $\begin{array}{c}\text { Sessões que } \\
\text { totalizavam } 10 \\
\text { horas de contato } \\
\text { em sala de aula }\end{array}$ & $\begin{array}{l}\text { O curso de humanidades médicas foi } \\
\text { uma matéria optativa. Antes de cada } \\
\text { sessão os alunos recebiam textos para } \\
\text { leitura prévia. Os principais tópicos } \\
\text { abordados foram a importância da } \\
\text { cultura e das relações sociais e a } \\
\text { história da medicina. Todas as aulas } \\
\text { tiveram como atividade dinâmica a } \\
\text { interpretação e discussão de artes } \\
\text { visuais e/ou literatura. }\end{array}$ & $\begin{array}{l}\text { Tanto o grupo da } \\
\text { intervenção } \\
\text { quanto o controle } \\
\text { completaram a } \\
\text { JSE-S no início e } \\
\text { no final do estudo }\end{array}$ & $\begin{array}{c}\text { Sim, aumento da } \\
\text { empatia } \\
\text { imediatamente após } \\
\text { intervenção }\end{array}$ \\
\hline
\end{tabular}




\begin{tabular}{|c|c|c|c|c|c|c|c|}
\hline Autor e Título & $\begin{array}{l}\text { Ano e país } \\
\text { da } \\
\text { publicação }\end{array}$ & $\begin{array}{l}\text { Participantes } \\
\text { do estudo }\end{array}$ & $\begin{array}{l}\text { Intervenção } \\
\text { opcional } \\
\text { /obrigatória }\end{array}$ & $\begin{array}{l}\text { Duração da } \\
\text { intervenção }\end{array}$ & Intervenção/Tipo da intervenção* & $\begin{array}{c}\text { Instrumento de } \\
\text { medida/ avaliação } \\
\text { da empatia }\end{array}$ & $\begin{array}{c}\text { Autores reportaram um } \\
\text { aumento significativo da } \\
\text { empatia? }\end{array}$ \\
\hline $\begin{array}{l}\text { Kaplan-Liss E, et } \\
\text { al. (2018) } \\
\\
\text { Teaching Medical } \\
\text { Students to } \\
\text { Communicate } \\
\text { With Empathy and } \\
\text { Clarity Using } \\
\text { Improvisation. }\end{array}$ & $\begin{array}{l}2018 \\
\text { EUA }\end{array}$ & $\begin{array}{c}114 \\
\text { estudantes } \\
\text { de medicina, } \\
\text { enfermagem } \\
\text { e odontologia }\end{array}$ & Opcional & $\begin{array}{l}\text { Curso composto } \\
\text { por seis sessões } \\
\text { com duração de } \\
\text { três horas cada }\end{array}$ & $\begin{array}{l}\text { Curso de ciências comunicativas como } \\
\text { matéria optativa. Consistiu de seis } \\
\text { sessões para abordar técnicas de } \\
\text { comunicação, sendo elas: (1) } \\
\text { Improvisação para cientistas: ensina } \\
\text { os futuros profissionais da saúde a se } \\
\text { comunicarem utilizando técnicas de } \\
\text { teatro improvisadas; (2) Aprimorando } \\
\text { sua mensagem: ensina técnicas de } \\
\text { "contar história". (3) Comunicando com } \\
\text { a comunidade: utiliza técnicas que } \\
\text { associam competência cultural e } \\
\text { educação em saúde para ensinar a } \\
\text { adquirir engajamento a população; (4) } \\
\text { Entrevistas: prática das técnicas } \\
\text { aprendidas por meio de gravações de } \\
\text { entrevistas. Essas foram revisadas e } \\
\text { tiveram feedback para os alunos de } \\
\text { jornalistas treinados; (5) Principais } \\
\text { públicos: Práticas dos conhecimentos } \\
\text { aprendidos com diferentes públicos; e } \\
\text { (6) Interpretação de interações com } \\
\text { pacientes: simulação de relações } \\
\text { médico-paciente satisfatórias, baseada } \\
\text { nas ferramentas de comunicação } \\
\text { aprendidas. }\end{array}$ & $\begin{array}{l}\text { Instrumento do } \\
\text { próprio autor }\end{array}$ & $\begin{array}{l}\text { Sim, o curso aumentou } \\
\text { o comportamento } \\
\text { empático dos alunos e } \\
\text { foi comprovada a } \\
\text { melhor aceitação por } \\
\text { parte dos alunos às } \\
\text { estratégias de } \\
\text { improvisação. Porém, } \\
\text { esse resultado não foi } \\
\text { avaliado a longo } \\
\text { prazo. }\end{array}$ \\
\hline
\end{tabular}




\begin{tabular}{|c|c|c|c|c|c|c|c|}
\hline Autor e Título & $\begin{array}{l}\text { Ano e país } \\
\text { da } \\
\text { publicação }\end{array}$ & $\begin{array}{l}\text { Participantes } \\
\text { do estudo }\end{array}$ & $\begin{array}{l}\text { Intervenção } \\
\text { opcional } \\
\text { /obrigatória }\end{array}$ & $\begin{array}{l}\text { Duração da } \\
\text { intervenção }\end{array}$ & Intervenção/Tipo da intervenção* & $\begin{array}{l}\text { Instrumento de } \\
\text { medida/ avaliação } \\
\text { da empatia }\end{array}$ & $\begin{array}{c}\text { Autores reportaram um } \\
\text { aumento significativo da } \\
\text { empatia? }\end{array}$ \\
\hline $\begin{array}{c}\text { Shapiro J, et al. } \\
\text { (2019) } \\
\text { The Human } \\
\text { Kindness } \\
\text { Curriculum: An } \\
\text { Innovative } \\
\text { Preclinical } \\
\text { Initiative to } \\
\text { Highlight } \\
\text { Kindness and } \\
\text { Empathy in } \\
\text { Medicine. }\end{array}$ & $\begin{array}{l}2019 \\
\text { EUA }\end{array}$ & $\begin{array}{c}343 \\
\text { estudantes } \\
\text { de medicina } \\
\text { do } 1^{\circ} \text { e } 2^{\circ} \\
\text { ano do curso }\end{array}$ & Obrigatória & $\begin{array}{l}\text { Quatro aulas, } \\
\text { cada uma com } \\
\text { duração de } 90 \\
\text { minutos }\end{array}$ & $\begin{array}{l}\text { Programa de estudos que era feito por } \\
\text { meio de } 4 \text { aulas, que abordavam } 6 \\
\text { áreas: (1) em relação à arte e a } \\
\text { humanização, que incluía aulas de } \\
\text { teatro; (2) habilidades médicas de } \\
\text { tomada de perspectiva e atenção na } \\
\text { linguagem; (3) neurociências com foco } \\
\text { em aumentar a compreensão dos } \\
\text { neurônios espelho e outros aspectos } \\
\text { da regulação neuronal de emoções; } \\
\text { (4) meditação com foco em promover } \\
\text { aspectos de compaixão e também } \\
\text { para reduzir a ansiedade entre os } \\
\text { alunos; (5) interação com "pacientes- } \\
\text { padrão" virtuais; (6) utilização de } \\
\text { vídeos que abordam o comportamento } \\
\text { da empatia, elaborados por Riess et al. } \\
\text { (Harvard).O programa foi dividido em } 2 \\
\text { anos, sendo o primeiro o ano piloto, } \\
\text { nos quais os alunos de ambos os } \\
\text { grupos (1 e } 2 \text { ) receberam as mesmas } \\
\text { aulas. No ano seguinte } \\
\text { (implementação), os grupos } 3 \text { e } 4 \\
\text { receberam aulas diferentes, sendo que } \\
\text { o grupo } 3 \text { contou com o mesmo } \\
\text { programa do ano piloto. }\end{array}$ & $\begin{array}{c}\text { JSE-S e } \\
\text { avaliações } \\
\text { individuais depois } \\
\text { das aulas, } \\
\text { utilizando uma } \\
\text { escala de } 5 \\
\text { pontos (escala de } \\
\text { Likert), abordando } \\
\text { três aspectos: } \\
\text { impacto da } \\
\text { sessão no } \\
\text { desenvolvimento } \\
\text { do aluno como } \\
\text { médico; } \\
\text { apropriação dos } \\
\text { métodos de } \\
\text { ensino e } \\
\text { relevância para o } \\
\text { currículo médico. } \\
\text { Além disso, } \\
\text { opiniões } \\
\text { narrativas foram } \\
\text { solicitadas e } \\
\text { gravadas. }\end{array}$ & $\begin{array}{c}\text { Sim, no ano de } \\
\text { implementação, houve } \\
\text { uma melhora } \\
\text { principalmente nos } \\
\text { alunos que já tinham } \\
\text { inicialmente níveis } \\
\text { baixos de empatia, } \\
\text { naqueles com mais } \\
\text { idade e em mulheres. } \\
\text { E, por meio de um } \\
\text { acompanhamento } \\
\text { longitudinal dos alunos } \\
\text { do estudo piloto, foi } \\
\text { possível observar que } \\
\text { os níveis de empatia } \\
\text { não sofreram } \\
\text { decréscimo nos anos } \\
\text { seguintes, incluindo o } \\
\text { 3o ano do curso (onde } \\
\text { a literatura aponta } \\
\text { para queda } \\
\text { significativa). }\end{array}$ \\
\hline
\end{tabular}




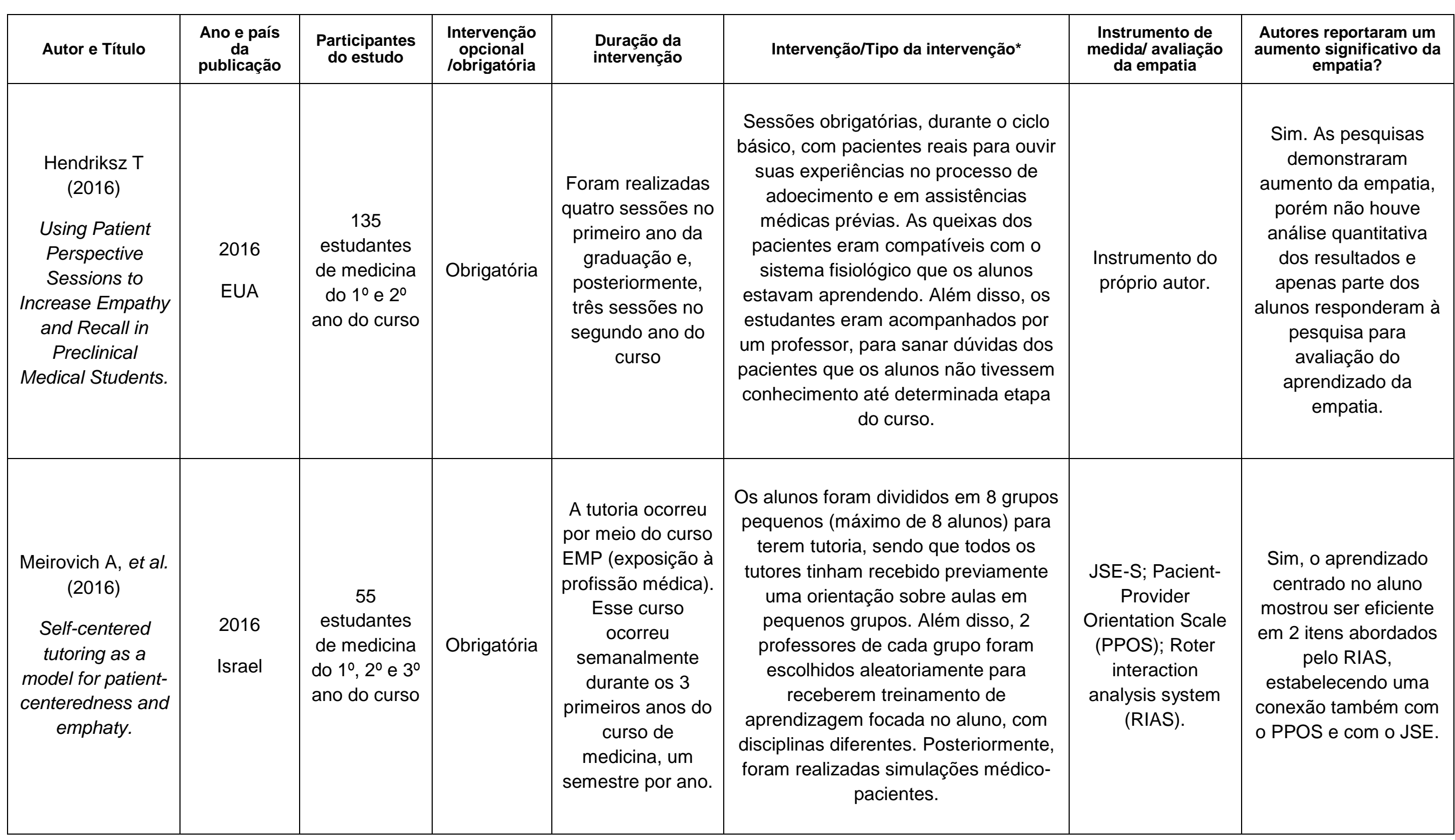




\begin{tabular}{|c|c|c|c|c|c|c|c|}
\hline Autor e Título & $\begin{array}{l}\text { Ano e país } \\
\text { da } \\
\text { publicação }\end{array}$ & $\begin{array}{l}\text { Participantes } \\
\text { do estudo }\end{array}$ & $\begin{array}{l}\text { Intervenção } \\
\text { opcional } \\
\text { /obrigatória }\end{array}$ & $\begin{array}{l}\text { Duração da } \\
\text { intervenção }\end{array}$ & Intervenção/Tipo da intervenção* & $\begin{array}{l}\text { Instrumento de } \\
\text { medida/ avaliação } \\
\text { da empatia }\end{array}$ & $\begin{array}{c}\text { Autores reportaram um } \\
\text { aumento significativo da } \\
\text { empatia? }\end{array}$ \\
\hline $\begin{array}{l}\text { Sweeney K e } \\
\text { Baker P (2018) } \\
\text { Promoting } \\
\text { empathy using } \\
\text { video-based } \\
\text { teaching. }\end{array}$ & $\begin{array}{l}2017 \\
\text { Reino } \\
\text { Unido }\end{array}$ & $\begin{array}{c}48 \\
\text { estudantes } \\
\text { de medicina } \\
\text { do } 1^{\circ} \text { e } 2^{\circ} \\
\text { ano do curso }\end{array}$ & Obrigatória & $\begin{array}{l}\text { Uma sessão de } \\
\text { ensino com } \\
\text { duração de } 90 \\
\text { minutos }\end{array}$ & $\begin{array}{l}\text { Uma aula com vídeos de relatos de } \\
\text { pacientes reais (10 a } 12 \text { min), em } \\
\text { formato de entrevista semiestruturada, } \\
\text { em que os pacientes falavam sobre } \\
\text { experiências em hospitais e de } \\
\text { interações com médicos. Foram } \\
\text { citados exemplos de momentos em } \\
\text { que a comunicação médico-paciente } \\
\text { foi inadequada. Posteriormente, } \\
\text { realizava-se uma reflexão em } \\
\text { pequenos grupos acerca das situações } \\
\text { demonstradas nos vídeos. }\end{array}$ & $\begin{array}{l}\text { Instrumento do } \\
\text { próprio autor e } \\
\text { PPOS. }\end{array}$ & $\begin{array}{l}\text { Sim, houve aumento } \\
\text { na empatia obtido por } \\
\text { meio de relatos dos } \\
\text { alunos e avaliado pelo } \\
\text { PPOS comparando } \\
\text { análise pré e pós- } \\
\text { sessões. Porém não } \\
\text { houve avaliação a } \\
\text { longo prazo. }\end{array}$ \\
\hline $\begin{array}{l}\text { Ahmadzadeh A, } \\
\text { et al. (2019) } \\
\text { Does watching a } \\
\text { movie improve } \\
\text { empathy? A } \\
\text { cluster } \\
\text { randomized } \\
\text { controlled trial. }\end{array}$ & $\begin{array}{l}2019 \\
\text { Irã }\end{array}$ & $\begin{array}{c}133 \\
\text { estudantes } \\
\text { de medicina } \\
\text { do } 4^{\circ} \text { ao } 7^{\circ} \\
\text { período }\end{array}$ & Opcional & $\begin{array}{c}\text { Ambos foram } \\
\text { intervenções } \\
\text { únicas, filme e } \\
\text { workshop de } 3 \\
\text { horas de duração }\end{array}$ & $\begin{array}{c}\text { Os estudantes foram divididos em } 4 \\
\text { grupos que tiveram diferentes } \\
\text { intervenções: } \\
\text { 1.Grupo A: Workshop de habilidades } \\
\text { de comunicação por três horas } \\
\text { (incluindo perguntas sobre as } \\
\text { experiências pessoais de cada um na } \\
\text { medicina e discussões sobre conceitos } \\
\text { como empatia e relação entre médico } \\
\text { e paciente) } \\
\text { 2.Grupo B: assistiu filme "The Doctor" } \\
\text { (Sobre a experiência de um cirurgião } \\
\text { cardiovascular ao ser diagnosticado } \\
\text { com câncer: } \\
\text { 3.Grupo C: realizando ambas as } \\
\text { atividades (filme e workshop) } \\
\text { 4. Grupo D: controle - nenhuma } \\
\text { intervenção específica. }\end{array}$ & $\begin{array}{c}\text { JSE-S foi } \\
\text { aplicada nos } 4 \\
\text { grupos em } 3 \\
\text { situações: antes, } \\
\text { imediatamente } \\
\text { após e um mês } \\
\text { depois da } \\
\text { intervenção. }\end{array}$ & $\begin{array}{c}\text { Sim, aumento de } \\
\text { empatia } \\
\text { imediatamente após as } \\
\text { intervenções, que se } \\
\text { sustentou após um } \\
\text { mês, no grupo A } \\
\text { (somente workshop) e } \\
\text { no grupo C (filme e } \\
\text { workshop). }\end{array}$ \\
\hline
\end{tabular}




\begin{tabular}{|c|c|c|c|c|c|c|c|}
\hline Autor e Título & $\begin{array}{c}\text { Ano e país } \\
\text { da } \\
\text { publicação }\end{array}$ & $\begin{array}{l}\text { Participantes } \\
\text { do estudo }\end{array}$ & $\begin{array}{l}\text { Intervenção } \\
\text { opcional } \\
\text { /obrigatória }\end{array}$ & $\begin{array}{l}\text { Duração da } \\
\text { intervenção }\end{array}$ & Intervenção/Tipo da intervenção* & $\begin{array}{c}\text { Instrumento de } \\
\text { medida/ avaliação } \\
\text { da empatia }\end{array}$ & $\begin{array}{c}\text { Autores reportaram um } \\
\text { aumento significativo da } \\
\text { empatia? }\end{array}$ \\
\hline $\begin{array}{l}\text { Ruiz-Moral R, et } \\
\text { al. (2017) } \\
\text { Teaching medical } \\
\text { students to } \\
\text { express empathy } \\
\text { by exploring } \\
\text { patient emotions } \\
\text { and experiences } \\
\text { in standardized } \\
\text { medical } \\
\text { encounters. }\end{array}$ & $\begin{array}{c}2017 \\
\text { Espanha }\end{array}$ & $\begin{array}{c}115 \\
\text { estudantes } \\
\text { de medicina } \\
\text { do } 3^{\circ} \text { ano do } \\
\text { curso }\end{array}$ & Obrigatória & $\begin{array}{l}\text { Curso com } \\
\text { duração de seis } \\
\text { semanas. }\end{array}$ & $\begin{array}{l}\text { Foi realizada uma matéria obrigatória, } \\
\text { que consistiu em cinco atividades: } \\
\text { 1) uma discussão em grupo sobre } \\
\text { vídeos e casos clínicos; 2) o } \\
\text { preenchimento de um formulário } \\
\text { eletrônico relatando experiências } \\
\text { pessoais de doenças vividas no } \\
\text { passado; 3) um workshop com } \\
\text { pacientes simulados (SPs); 4) uma } \\
\text { encenação entre os alunos com as } \\
\text { experiências coletadas no formulário; } \\
\text { 5) realização de entrevistas gravadas } \\
\text { entre os alunos e SPs. Houve } \\
\text { feedback ao final do curso. }\end{array}$ & $\begin{array}{l}\text { Instrumento do } \\
\text { próprio autor } \\
\text { aplicado apenas } \\
\text { ao final do curso }\end{array}$ & $\begin{array}{c}\text { Sim, o } \\
\text { desenvolvimento de } \\
\text { habilidades de } \\
\text { comunicação parece } \\
\text { aumentar o } \\
\text { comportamento } \\
\text { empático dos alunos } \\
\text { na relação médico- } \\
\text { paciente. }\end{array}$ \\
\hline
\end{tabular}




\begin{tabular}{|c|c|c|c|c|c|c|c|}
\hline Autor e Título & $\begin{array}{l}\text { Ano e país } \\
\text { da } \\
\text { publicação }\end{array}$ & $\begin{array}{l}\text { Participantes } \\
\text { do estudo }\end{array}$ & $\begin{array}{l}\text { Intervenção } \\
\text { opcional } \\
\text { /obrigatória }\end{array}$ & $\begin{array}{l}\text { Duração da } \\
\text { intervenção }\end{array}$ & Intervenção/Tipo da intervenção* & $\begin{array}{l}\text { Instrumento de } \\
\text { medida/ avaliação } \\
\text { da empatia }\end{array}$ & $\begin{array}{l}\text { Autores reportaram um } \\
\text { aumento significativo da } \\
\text { empatia? }\end{array}$ \\
\hline $\begin{array}{l}\text { Kataoka } \mathrm{H} \text {, et al. } \\
\text { (2019) } \\
\text { Can } \\
\text { communication } \\
\text { skills training } \\
\text { improve } \\
\text { empathy? A six- } \\
\text { year longitudinal } \\
\text { study of medical } \\
\text { students in Japan. }\end{array}$ & $\begin{array}{l}2018 \\
\text { Japão }\end{array}$ & $\begin{array}{c}116 \\
\text { estudantes } \\
\text { de medicina } \\
\text { do } 3^{\circ} \text { e } 4^{\circ} \\
\text { ano do curso }\end{array}$ & Obrigatório & $\begin{array}{l}\text { Workshop único } \\
\text { com duração de } \\
\text { quatro horas. }\end{array}$ & $\begin{array}{l}\text { Workshop único, obrigatório com } \\
\text { duração de } 4 \text { horas consistindo em: } \\
\text { aula teórica sobre comunicação e } \\
\text { entrevista médica; encenação de } \\
\text { consultas dos alunos com pacientes } \\
\text { simulados e discussões e feedback } \\
\text { sobre as consultas e o workshop }\end{array}$ & $\begin{array}{l}\text { JSE-S aplicado } \\
\text { no início do curso, } \\
\text { imediatamente } \\
\text { antes e } \\
\text { imediatamente } \\
\text { após o workshop } \\
\text { e no 5 e 6o ano } \\
\text { do curso (após } \\
\text { dois anos da } \\
\text { intervenção) }\end{array}$ & $\begin{array}{l}\text { Sim, aumento de } \\
\text { empatia } \\
\text { imediatamente após } \\
\text { intervenção. Entretanto } \\
\text { esse aumento não foi } \\
\text { sustentado (após dois } \\
\text { anos). }\end{array}$ \\
\hline $\begin{array}{c}\text { D'souza PC, et al. } \\
\text { (2020) } \\
\text { Effect of a Single- } \\
\text { Session } \\
\text { Communication } \\
\text { SkillsTraining on } \\
\text { Empathy in } \\
\text { Medical Students. }\end{array}$ & $\begin{array}{l}2019 \\
\text { Índia }\end{array}$ & $\begin{array}{c}80 \\
\text { estudantes } \\
\text { de medicina } \\
\text { do } 2^{\circ} \text { ano do } \\
\text { curso }\end{array}$ & Opcional & $\begin{array}{l}\text { Workshop único } \\
\text { com duração de } \\
\text { duas horas. }\end{array}$ & $\begin{array}{l}\text { Workshop único de duas horas sobre } \\
\text { habilidades de comunicação e } \\
\text { empatia. Foi realizada uma aula } \\
\text { teórica por PowerPoint, em que se } \\
\text { abordou o conceito da empatia e } \\
\text { formas verbais e não verbais de } \\
\text { demonstração empática. Depois, os } \\
\text { alunos assistiram vídeos e simulações } \\
\text { de consulta realizada pelos } \\
\text { professores, no qual os participantes } \\
\text { deveriam avaliar as técnicas de } \\
\text { comunicação usadas. Posteriormente, } \\
\text { houve uma encenação de consulta } \\
\text { onde os alunos fizeram o papel de } \\
\text { médico e os professores de paciente. } \\
\text { Ao final foi realizada uma reflexão } \\
\text { sobre as emoções do paciente durante } \\
\text { as consultas. }\end{array}$ & $\begin{array}{c}\text { JSE-S foi } \\
\text { aplicada em um } \\
\text { grupo controle e } \\
\text { grupo intervenção } \\
\text { (divididos de } \\
\text { forma } \\
\text { randomizada), em } \\
\text { três momentos: } \\
\text { antes, } \\
\text { imediatamente } \\
\text { após e três } \\
\text { semanas após o } \\
\text { curso. }\end{array}$ & $\begin{array}{l}\text { Sim, aumento de } \\
\text { empatia } \\
\text { imediatamente após } \\
\text { intervenção. Entretanto } \\
\text { esse aumento não foi } \\
\text { sustentado (após três } \\
\text { semanas). }\end{array}$ \\
\hline
\end{tabular}




\begin{tabular}{|c|c|c|c|c|c|c|c|}
\hline Autor e Título & $\begin{array}{l}\text { Ano e país } \\
\text { da } \\
\text { publicação }\end{array}$ & $\begin{array}{l}\text { Participantes } \\
\text { do estudo }\end{array}$ & $\begin{array}{l}\text { Intervenção } \\
\text { opcional } \\
\text { /obrigatória }\end{array}$ & $\begin{array}{l}\text { Duração da } \\
\text { intervenção }\end{array}$ & Intervenção/Tipo da intervenção* & $\begin{array}{l}\text { Instrumento de } \\
\text { medida/ avaliação } \\
\text { da empatia }\end{array}$ & $\begin{array}{c}\text { Autores reportaram um } \\
\text { aumento significativo da } \\
\text { empatia? }\end{array}$ \\
\hline $\begin{array}{l}\text { Wündrich } \mathrm{M} \text {, et al. } \\
\text { (2017) } \\
\text { Empathy training } \\
\text { in medical } \\
\text { students - a } \\
\text { randomized } \\
\text { controlled trial }\end{array}$ & $\begin{array}{c}2017 \\
\text { Alemanha }\end{array}$ & $\begin{array}{c}158 \\
\text { estudantes } \\
\text { de medicina } \\
\text { do } 3^{\circ} \text { ano do } \\
\text { curso }\end{array}$ & Obrigatório & $\begin{array}{c}\text { Curso com } \\
\text { duração de três } \\
\text { semanas, com } \\
\text { duas aulas } \\
\text { teóricas com } \\
\text { duração de } 2 \mathrm{~h} \text { e } \\
25 \text { minutos cada, } \\
\text { e com a } \\
\text { simulação de } \\
\text { consulta realizada } \\
\text { na última semana, } \\
\text { com duração de } \\
10 \text { minutos cada } \\
\text { simulação. }\end{array}$ & $\begin{array}{l}\text { Foi realizada um curso obrigatório } \\
\text { durante a disciplina de psiquiatria. Os } \\
\text { alunos deveriam: refletir sobre as } \\
\text { próprias experiências com pacientes e } \\
\text { sobre a empatia na relação médico- } \\
\text { paciente; identificar comportamentos } \\
\text { empáticos em consultas simuladas } \\
\text { realizadas pelos professores e realizar } \\
\text { entrevistas médicas com pacientes } \\
\text { psiquiátricos simulados, recebendo } \\
\text { feedback de outro aluno e dos SPs, } \\
\text { além de auto avaliar seu } \\
\text { comportamento durante a consulta. }\end{array}$ & $\begin{array}{l}\text { Instrumento do } \\
\text { próprio autor e } \\
\text { JSE-S que foram } \\
\text { aplicadas em } \\
\text { grupo controle e } \\
\text { grupo } \\
\text { intervenção. }\end{array}$ & $\begin{array}{l}\text { Sim, eles relatam que } \\
\text { apesar de não ter sido } \\
\text { encontrado diferenças } \\
\text { entre as escalas de } \\
\text { JSE do grupo controle } \\
\text { e intervenção, os } \\
\text { pacientes simulados } \\
\text { sentiram um } \\
\text { comportamento mais } \\
\text { empático no grupo que } \\
\text { realizou o curso. }\end{array}$ \\
\hline $\begin{array}{c}\text { Chen A, et al. } \\
(2018) \\
\text { Teaching } \\
\text { Empathy: the } \\
\text { Implementation of } \\
\text { a Video Game } \\
\text { into a Psychiatry } \\
\text { Clerkship } \\
\text { Curriculum. }\end{array}$ & $\begin{array}{l}2017 \\
\text { EUA }\end{array}$ & $\begin{array}{c}84 \\
\text { estudantes } \\
\text { de medicina } \\
\text { do } 3^{\circ} \text { ano do } \\
\text { curso, que } \\
\text { estavam } \\
\text { iniciando } \\
\text { estágios em } \\
\text { psiquiatria. }\end{array}$ & $\begin{array}{c}\text { Não } \\
\text { esclarecido }\end{array}$ & Intervenção única. & $\begin{array}{l}\text { O vídeo game "That Dragon, Cancer" } \\
\text { conta a história de uma criança que foi } \\
\text { diagnosticada com um tumor aos doze } \\
\text { meses de idade e falece aos } 5 \text { anos. } \\
\text { Nele, o jogador assume o papel dos } \\
\text { pais da criança, sendo possível viver } \\
\text { momentos durante o adoecimento, } \\
\text { escutar áudios com narrativas dos } \\
\text { pais, entre outros. Esses momentos } \\
\text { são intercalados com períodos de } \\
\text { diversão, por exemplo, uma corrida } \\
\text { que acontece ao redor do hospital. O } \\
\text { jogo tem o objetivo de possibilitar que } \\
\text { o estudante de medicina viva a } \\
\text { experiência do paciente e da família,e } \\
\text { dessa maneira sensibilizá-los para a } \\
\text { perspectiva do paciente. }\end{array}$ & $\begin{array}{l}\text { O JSE-S foi } \\
\text { aplicado antes } \\
\text { dos alunos serem } \\
\text { orientados à jogar } \\
\text { o "That Dragon, } \\
\text { Cancer", além de } \\
\text { responderem } \\
\text { perguntas } \\
\text { realizadas por } \\
\text { meio de um } \\
\text { instrumento do } \\
\text { próprio autor, que } \\
\text { foi aplicado } \\
\text { posteriormente ao } \\
\text { jogo, com } \\
\text { algumas } \\
\text { alterações. }\end{array}$ & $\begin{array}{l}\text { Sim, a utilização do } \\
\text { videogame como } \\
\text { ferramenta de ensino } \\
\text { apresentou impacto } \\
\text { positivo no aumento } \\
\text { da empatia } \\
\text { imediatamente após a } \\
\text { intervenção. Alguns } \\
\text { alunos ainda } \\
\text { reforçaram que essa } \\
\text { estratégia pode ser } \\
\text { mais didática que } \\
\text { métodos tradicionais } \\
\text { de ensino }\end{array}$ \\
\hline
\end{tabular}




\begin{tabular}{|c|c|c|c|c|c|c|c|}
\hline Autor e Título & $\begin{array}{l}\text { Ano e país } \\
\text { da } \\
\text { publicação }\end{array}$ & $\begin{array}{l}\text { Participantes } \\
\text { do estudo }\end{array}$ & $\begin{array}{l}\text { Intervenção } \\
\text { opcional } \\
\text { /obrigatória }\end{array}$ & $\begin{array}{l}\text { Duração da } \\
\text { intervenção }\end{array}$ & Intervenção/Tipo da intervenção* & $\begin{array}{l}\text { Instrumento de } \\
\text { medida/ avaliação } \\
\text { da empatia }\end{array}$ & $\begin{array}{c}\text { Autores reportaram um } \\
\text { aumento significativo da } \\
\text { empatia? }\end{array}$ \\
\hline $\begin{array}{c}\text { Foster A, et al. } \\
\text { (2016) } \\
\text { Using Virtual } \\
\text { Patients to Teach } \\
\text { Empathy: } A \\
\text { Randomized } \\
\text { Controlled Study } \\
\text { to Enhance } \\
\text { Medical Students' } \\
\text { Empathic } \\
\text { Communication. }\end{array}$ & $\begin{array}{l}2016 \\
\text { EUA }\end{array}$ & $\begin{array}{c}70 \\
\text { estudantes } \\
\text { de medicina } \\
\text { do } 1^{\circ} \text { ano do } \\
\text { curso }\end{array}$ & $\begin{array}{l}\text { Opcional } \\
\text { (alunos } \\
\text { foram } \\
\text { contatados } \\
\text { e } \\
\text { receberam } \\
\text { auxílio de } \\
\$ 20 \text { pelo } \\
\text { tempo e } \\
\text { recursos } \\
\text { gastos com } \\
\text { viagens). }\end{array}$ & Única & $\begin{array}{l}\text { Os alunos interagiram com uma paciente } \\
\text { virtual (PV) de } 21 \text { anos com sintomas de } \\
\text { depressão maior, sendo coletada a } \\
\text { entrevista por meio digital, com diálogos } \\
\text { digitados. Houve divisão dos participantes } \\
\text { em três grupos e cada grupo interagiu com } \\
\text { um dos três cenários disponibilizados, } \\
\text { sendo eles: PV com depressão, patient } \\
\text { shadowing (relata seu histórico e } \\
\text { inseguranças que isso gera em uma nova } \\
\text { consulta) e PV que, ao final da consulta, dá } \\
\text { um retorno acerca do sentimento que teve } \\
\text { durante o atendimento e diantes das } \\
\text { atitudes empáticas do médico. Após essa } \\
\text { intervenção, os alunos interagiram com } \\
\text { pacientes padronizados (atores treinados) } \\
\text { no mesmo cenário com a finalidade de } \\
\text { entender se há uma percepção muito } \\
\text { distinta entre a maneira como o caso é } \\
\text { conduzido utilizando pacientes } \\
\text { padronizados e virtuais. }\end{array}$ & $\begin{array}{l}\text { Instrumento do } \\
\text { próprio autor. }\end{array}$ & $\begin{array}{c}\text { Sim, há um aumento } \\
\text { geral da empatia com } \\
\text { melhores respostas às } \\
\text { oportunidades } \\
\text { empáticas quando } \\
\text { possuem um feedback } \\
\text { imediato, porém } \\
\text { apenas algumas } \\
\text { associações foram } \\
\text { estatisticamente } \\
\text { significativas. }\end{array}$ \\
\hline $\begin{array}{c}\text { Peixoto JM e } \\
\text { Moura EP (2020) } \\
\text { Mapa da Empatia } \\
\text { em Saúde: } \\
\text { Elaboração de um } \\
\text { instrumento para } \\
\text { o } \\
\text { Desenvolvimento } \\
\text { da Empatia }\end{array}$ & $\begin{array}{l}2020 \\
\text { Brasil }\end{array}$ & $\begin{array}{c}20 \\
\text { estudantes } \\
\text { de medicina } \\
\text { do } 3^{\circ} \text { ano do } \\
\text { curso }\end{array}$ & $\begin{array}{l}\text { Não se } \\
\text { aplica }\end{array}$ & Não se aplica & $\begin{array}{l}\text { O Mapa da Empatia em Saúde (MES) é } \\
\text { uma ferramenta utilizada para captar o } \\
\text { nível de empatia atingido durante uma } \\
\text { consulta médica e para estimular uma } \\
\text { reflexão por parte do estudante de } \\
\text { medicina acerca de suas atitudes e falas } \\
\text { empáticas. Nele, o aluno deve responder: } \\
\text { (1) "O que você sentiria se estivesse no } \\
\text { lugar desta pessoa?" (2) " Qual a sua } \\
\text { percepção das necessidades e desejos } \\
\text { dessa pessoa, atuais e futuras?" (3) "Como } \\
\text { me sinto sentindo a história dessa } \\
\text { pessoa?" (4) "Como posso ajudar esta } \\
\text { pessoa?". Após o preenchimento, há a } \\
\text { instrução para escolher o "rosto"'" que } \\
\text { melhor expresse os sentimentos da pessoa } \\
\text { percebidos pelo aluno. }\end{array}$ & $\begin{array}{l}\text { Instrumento do } \\
\text { próprio autor. }\end{array}$ & $\begin{array}{c}\text { Sim, instrumento foi } \\
\text { classificado como uma } \\
\text { ferramenta promissora. } \\
\text { Um estudo que } \\
\text { objetiva avaliar os } \\
\text { resultados do uso } \\
\text { deste instrumento se } \\
\text { encontra em } \\
\text { andamento. }\end{array}$ \\
\hline
\end{tabular}

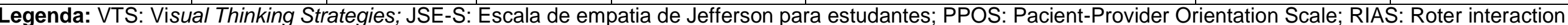
analysis system; PV: pacientes virtuais; SP: paciente simulados.

Fonte: Moura EP, et al., 2021. 


\section{DISCUSSÃO}

Esta revisão sugere a importância da utilização de estratégias voltadas para o desenvolvimento dos aspectos subjetivos do atendimento clínico no desenvolvimento da empatia durante a formação dos acadêmicos de medicina. A compreensão profunda e sem julgamentos, por meio de um "ver" e "ouvir" característicos da empatia faz-se necessária na educação médica. Nos últimos anos, na perspectiva da medicina centrada no paciente, a comunicação empática assume um papel importante na graduação médica, visto que essa habilidade corrobora em uma melhor relação médico-paciente (RIBEIRO, 2008; SULLIVAN M, 2003).

Assim, o treinamento dos estudantes para a percepção do aspecto científico e empírico da doença faz-se fundamental (ROSSI SP, 2006). Nossos resultados demonstraram que, atualmente, semelhante ao relatado em revisões realizadas em períodos anteriores, as estratégias que vêm sendo utilizadas para o ensino da empatia incluem artes, cursos de treinamento de comunicação, contato com pacientes e reflexão, bem como a utilização da realidade virtual.

O uso da arte como ferramenta de ensino está relacionado ao aumento da empatia (GRAHAM J, 2016; AHMADZADEH A, 2019; BENTWICH ME, 2017; SHAPIRO J, 2019). Segundo Ahmadzadeh A, et al. (2019), o envolvimento emocional do estudante, estimulado pela arte, resulta em maior motivação e aproveitamento do aprendizado estruturado proporcionado pelo curso de comunicação, possibilitando um retardo no declínio da empatia ao longo do tempo. Tais achados corroboram com dados da literatura que relatam a possibilidade de uma rica experiência de aprendizagem a partir da natureza metafórica das artes. Isto ocorre por meio do desafio do pensamento concreto ou literal ao legitimar experiências e emoções pessoais dos alunos e aprimorando a tolerância às ambiguidades, com consequente redimensionamento da prática clínica (HAIDET $\mathrm{P}, 2016)$.

No que diz respeito à utilização da comunicação (KATAOKA H, 2019; AHMADZADEH A, 2019; SHAPIRO J, 2019; D'SOUZA PC, 2019; SCHWELLER M, 2017; KAPLAN-LISS E, 2018; MEIROVICH A, 2016; RUIZMORAL R, 2017; WÜNDRICH M, 2017), nossos resultados reforçam que cursos de comunicação são possíveis formas de se ensinar empatia ao possibilitarem o treinamento dos estudantes para a percepção dos dois aspectos da doença (ROSSI SP, 2006).

A boa qualidade da comunicação médico-paciente, que se caracteriza por uma escuta atenta, é essencial para o aprimoramento da prática clínica. $O$ uso da reflexão também se mostrou como uma ferramenta potencial para o ensino da empatia. Com o emprego do Mapa da Empatia em Saúde, por exemplo, os estudantes são estimulados a refletir sobre tomada de perspectiva, compreensão empática e preocupação empática no contexto de atendimento clínico, o que contribui para o aprendizado desse construto (Peixoto JM e Moura EP, 2020).

Por fim, no campo da tecnologia, dois trabalhos utilizaram realidade virtual, com jogos de videogames ou pacientes virtuais para o ensino da empatia Chen A, et al. (2018) e Foster A, et al. (2016). Estudos apontam um maior envolvimento e motivação dos alunos ao utilizarem videogames na educação médica (BOYLE EA, 2016). Segundo Foster A, et al. (2016), a utilização de pacientes virtuais permite: simular cenários clínicos, oferecer conteúdo padronizado, e possibilita maior tempo para o estudante pensar nas respostas dadas às suas perguntas. Dessa forma, as Tecnologias Digitais de Informação e Comunicação assumem um papel importante trazendo também a possibilidade para o ensino das habilidades socioemocionais (GORGENF, 2018).

Quanto à forma de implementação das estratégias de ensino da empatia no curso, observamos que, nos estudos selecionados, cinco a utilizaram de forma opcional, o que pode levar a um viés de seleção, uma vez que os estudantes que escolhem cursos de humanidades podem estar predispostos a resultados mais favoráveis de empatia. Além disso, na maioria dos estudos, as intervenções foram concentradas durante os três primeiros anos do curso, sendo que nenhum artigo ofereceu curso de comunicação após o 4 ano. Segundo Scheweller et al. (2017), as intervenções precoces, em especial durante o primeiro ano da graduação, são significativas para aumentar a empatia entre os estudantes e propiciar o desenvolvimento de uma relação médico-paciente mais empática. 
Entretanto, os estudos que abordaram workshop como uma intervenção pontual, destacaram a necessidade de intervenções sustentadas durante o curso, uma vez que apenas uma intervenção não parece ser efetiva para a manutenção da empatia (KATAOKA H, 2019; D'SOUZA PC, 2019). Dessa forma, para obter resultados mais favoráveis de aprendizado da empatia, é interessante que as estratégias de ensino sejam intencionais, precoces e sustentadas.

Durante à análise dos estudos selecionados encontramos algumas limitações. Alguns estudos basearam o sucesso da intervenção na opinião do estudante sobre a satisfação e as mudanças de atitudes. Grande parte dos trabalhos utilizou questionários auto respondidos que avaliam a atitude dos estudantes e somente um estudo avaliou mudança de comportamento dos estudantes. Além disso, vários estudos relataram a impossibilidade de utilizar um grupo controle o que impede descartar influências externas nos resultados encontrados. Ainda, os artigos utilizaram intervenções variadas, não considerando os componentes afetivos e cognitivos da empatia como objetivos de aprendizagem. A maioria dos artigos realizaram a avaliação dos níveis de empatia apenas pós-intervenção, entretanto, não se sabe se estes resultados se sustentam, uma vez que o ensino da empatia requer mudança comportamental, e, portanto, necessita repetição.

\section{CONSIDERAÇÕES FINAIS}

Nossos resultados sugerem que a empatia é um construto que pode ser ensinado. Devido à natureza multidimensional da empatia, estratégias que abordam tanto os seus aspectos emocionais, quanto cognitivos se mostraram mais eficazes em aumentar o nível de empatia em estudantes de medicina, bem como minimizar o seu decréscimo a longo prazo. Neste contexto, a utilização de métodos combinados pode ser benéfica para o ensino. Os resultados sugerem ainda que as estratégias para o ensino da empatia devem ser realizadas de forma longitudinal, e não pontuais, para garantir a consolidação do aprendizado. Ressalta-se que a utilização de ambiente virtual mostrou ser uma estratégia eficaz, resultado relevante diante das modificações no ensino observadas na atualidade. No entanto estudos adicionais são necessários para avaliar o uso da tecnologia no ensino da empatia durante a graduação médica.

\section{REFERÊNCIAS}

1. AHMADZADEH A, et al. Does watching a movie improve empathy? A cluster randomized controlled trial. Can Med Educ J. Canada, 2019; 10(4): 4-12.

2. ARISO JM. Enhancing second-order empathy in medical practice by supplementing patients' narratives with certainties. BMC Med Educ. Madrid, 2018; 18(1): 1-8.

3. BATISTA NA, LESSA SS. Aprendizagem da Empatia na Relação Médico-Paciente: um Olhar Qualitativo entre Estudantes do Internato de Escolas Médicas do Nordeste do Brasil. Rev Bras Educ Med. Brasil, 2019; 43(1): 349356.

4. BENTWICH ME, Gilbey P. More than visual literacy: Art and the enhancement of tolerance for ambiguity and empathy. BMC Med Educ. 2017; 17(1): 1-9.

5. BOYLE EA, et al. An update to the systematic literature review of empirical evidence of the impacts and outcomes of computer games and serious games. Comput Educ. Washington, 2016; 94: 178-192.

6. CAMPBELL T, et al. Too Much Experience: A Desensitization Bias in Emotional Perspective Taking. J Pers Soc Psychol. Washington, 2014; 106(2): 272-285.

7. CARDOSO V, et al. Systematic review of mixed methods: Method of research for the incorporation of evidence in nursing. Texto e Context Enferm. Brasil, 2019; 28: 1-12.

8. CHEN AK, et al. The effect of Mind Body Medicine course on medical student empathy: A pilot study. Med Educ Online. Washington, 2016; 21(1): 1-4.

9. CHEN A, et al. Teaching Empathy: the Implementation of a Video Game into a Psychiatry Clerkship Curriculum. Acad Psychiatry. 2018; 42(3): 362-265.

10. COTTA FCK, et al. Cultura, ensino e aprendizagem da empatia na educação médica: scoping review. Interface Comun Saúde, Educ. Botucatu, 2020; 24: 1-15.

11. D'SOUZA PC. Effect of a Single-Session Communication Skills Training on Empathy in Medical Students. Academic Psychiatry. 2019; 44: 289-294.

12. FERREIRA, AB. Aurélio: o minidicionário da língua portuguesa. Rio de Janeiro: Positivo, 2002.

13. FOSTER A, et al. Using Virtual Patients to Teach Empathy: A Randomized Controlled Study to Enhance Medical Students' Empathic Communication. Simul Healthc. Florida, 2016;11(3): 181-189.

14. GRAHAM J, et al. Medical Humanities Coursework Is Associated with Greater Measured Empathy in Medical Students. Am J Med [online]. Washington, 2016; 129(12): 1334-1337. 
15. GREITEMEYER T, et al. Playing Prosocial Video Games Increases Empathy and Decreases Schadenfreude. Emotion. 2010; 10(6): 796-802.

16. HAIDET $P$, et al. A guiding framework to maximise the power of the arts in medical education: a systematic review an metasynthesis. Med Educ. 2016; 50(30): 320-331.

17. HENDRIKSZ T. Using patient perspective sessions to increase empathy and recall in preclinical medical students. $J$ Am Osteopath Assoc. 2016; 116(10): 662-666.

18. HIGGINS JPT, THOMAS J. Cochrane Handbook for Systematic Reviews ofInterventions Version 5.1.0 [online]. Cochrane database syst. rev. 2011.

19. JEFFREY D, DOWNIE R. Empathy: can it be taught? J R Coll Physicians Edinb. Reino Unido, 2016; 46: 107-112.

20. KAPLAN-LISS E, et al. Teaching medical students to communicate with empathy and clarity using improvisation. Acad Med. 2018; 93(3): 440-443.

21. KATAOKA H, et al. Can communication skills training improve empathy? A six-year longitudinal study of medical students in Japan. Med Teach [online]. 2019; 41(2): 195-200.

22. KLEINSMITH A. Understanding empathy training with virtual patients. Comput Human Behav [online]. 2015; 52: 151158.

23. LAUGHEY W, et al. Twelve tips for teaching empathy using simulated patients. Med Teacher [online]. 2019; 41(8): 883-887.

24. MAIROT LTS, et al. As Artes na Educação Médica: Revisão Sistemática da Literatura. Rev. bras. duc. med. Brasília, 2019; 43(4): 54-64.

25. MEIROVICH A, et al. Student-centered tutoring as a model for patient-centeredness and empathy. Adv Med Educ Pract. 2016; 7: 423-428.

26. PEIXOTO JM, MOURA EP. Mapa da Empatia em Saúde: Elaboração de um Instrumento para o Desenvolvimento da Empatia. Rev Bras Educ Med. Brasil, 2020; 44(1): 1-8.

27. RATKA A. Empathy and the development of affective skills. Am J Pharm Educ. Nova lorque, 2018; 82(10): 11401143.

28. ROSSI PS, BATISTA NA. O ensino da comunicação na graduação em Medicina - uma abordagem. Interface Comunic., Saúde, Educ. Botucatu, 2006; 10(19): 93-102.

29. RUIZ-MORAL R, et al. Teaching medical students to express empathy by exploring patient emotions and experiences in standardized medical encounters. Patient Educ Couns. Espanha, 2017; 100(9): 1694-1700.

30. SAN-MARTÍN M, et al. Role of a semiotics-based curriculum in empathy enhancement: A longitudinal study in three dominican medical schools. Front Psychol. 2017; 8: 1-8.

31. SCHWELLER M, et al. Nurturing virtues of the medical profession: does it enhance medical students' empathy? Int $J$ Med Educ. 2017; 8: 262-267.

32. SHAPIRO J, et al. The Human Kindness Curriculum: An Innovative Preclinical Initiative to Highlight Kindness and Empathy in Medicine. Educ. Health. 2019; 32(2): 53-61.

33. SON D, et al. Communication skills training and the conceptual structure of empathy among medical students. Perspect Med Educ. Japão, 2018; 7(4): 264-271.

34. SUARTZ CV, et al. Avaliação de empatia em residentes de especialidades clínicas e cirúrgicas da Universidade Federal de São Paulo. Rev Bras Educ Med. Brasil, 2013; 37(3): 320-325.

35. SULLIVAN M. The new subjective medicine: Taking the patient's point of view on health care and health. Soc Sci Med. Washington, 2003; 56(7): 1595-1604.

36. SWEENEY K, BAKER P. Promoting empathy using video-based teaching. Clin Teach. Reino Unido, 2018; 15(4): 336340.

37. WÜNDRICH M, et al. Empathy training in medical students-a randomized controlled trial. Med Teach [online]. 2017; 39(10): 1096-1098. 